CERN-EP/99-100

15 July 1999

\title{
FRONT-END ELECTRONICS FOR DRIFT TUBES IN A HIGH-RATE ENVIRONMENT
}

\author{
W. Riegler ${ }^{a, 1}$, M. Aleksa ${ }^{a}$, M. Deile ${ }^{b}$, J. Dubbert $^{b}$, C.W. Fabjan ${ }^{a}$, \\ C. Gruhn ${ }^{c}$, N.P. Hessey ${ }^{b, 2}$, T. Sammer ${ }^{b, 3}$
}

\begin{abstract}
A front-end electronics readout for drift tubes in a high-rate environment is presented. This system allows us to encode several pieces of information (leading edge time, trailing edge time, signal charge and piled-up hits from multiple tracks) into a single readout channel that is presented to the TDC. The advantage of active baseline restoration compared to bipolar signal shaping is discussed.
\end{abstract}

${ }^{a}$ EP Division, CERN, CH-1211 Geneva 23, Switzerland.

${ }^{b}$ Ludwig-Maximilians-Universität München, 80539 München, Germany.

${ }^{c}$ Max-Planck-Institut für Physik, Föhringer Ring 6, 80805 München, Germany.

${ }^{1}$ Now at Harvard University, 42 Oxford Street, Cambridge MA 02138, USA.

2 Now at NIKHEF, Kruislaan 409, 1098 SJ Amsterdam, Netherlands.

${ }^{3}$ Now at Max-Planck-Institut für Physik, Föhringer Ring 6, 80805 München, Germany. 


\section{Introduction}

The muon spectrometer of ATLAS [1, 2], a general purpose experiment at the future Large Hadron Collider (LHC), will consist of about 370000 pressurized cylindrical drift tubes assembled into about 1200 chambers. The high background counting rates of up to $300 \mathrm{kHz}$ per wire require low gas gain in order to avoid space-charge and ageing effects. The low gas gain deteriorates the resolution due to time-slewing effects, and the high counting rates cause inefficiencies due to signal pile-up. The drift tube parameters are shown in Table 1.

Table 1

Drift tube parameters.

\begin{tabular}{|l|l|}
\hline Inner/Outer tube radius & $1.46 / 1.5 \mathrm{~cm}$ \\
Tube length & $2-6 \mathrm{~m}$ \\
Wire diameter & $50 \mu \mathrm{m} \mathrm{W} / \mathrm{Re} 93 / 7$ \\
Gas & $\mathrm{Ar} / \mathrm{N}_{2} / \mathrm{CH}_{4} 91 / 4 / 5$ at 3 bars absolute [3] \\
Gas gain & $2 \times 10^{4}(3270 \mathrm{~V})$ \\
Maximum drift time & $480 \mathrm{~ns}$ \\
Preamp peaking time & $15 \mathrm{~ns}$ \\
Discriminator threshold & 18 primary electrons \\
\hline
\end{tabular}

In addition, to the leading edge time, the drift tube signal contains additional information which can be used to improve the drift tube performance. Since the signal path is AC-coupled, the signal integrates to zero over long times resulting in baseline shift and baseline fluctuations at high rates. Bipolar signal shaping and unipolar shaping with active baseline restoration are the two schemes to deal with this problem. We will compare the performance of both of them.

\section{Information contained in the signal}

\subsection{Leading edge time}

Two typical drift tube signals are shown in Fig. 1. The measurement of the leading edge time provides the position measurement. The position resolution of the drift chamber depends on the detector physics parameters such as gas mixture, gas gain, diffusion etc., and also on the electronics parameters like the preamplifier peaking time and threshold setting. A detailed analysis of all these effects is presented in Ref. [4]. Typically, a position resolution of $\sigma<80 \mu \mathrm{m}$ can be achieved.

\subsection{Trailing edge time}

The trailing edge time of the signal has a fixed latency with respect to the muon crossing time since the trailing edge corresponds to electrons created close to the tube wall (Fig. 1). The trailing edge time can be used offline to reject out-of-time events from uncorrelated background hits. The trailing edge time resolution depends on the tail cancellation scheme. Using standard unipolar shaping with a double pole/zero network [5] we achieved a resolution of $20 \mathrm{~ns}$. 


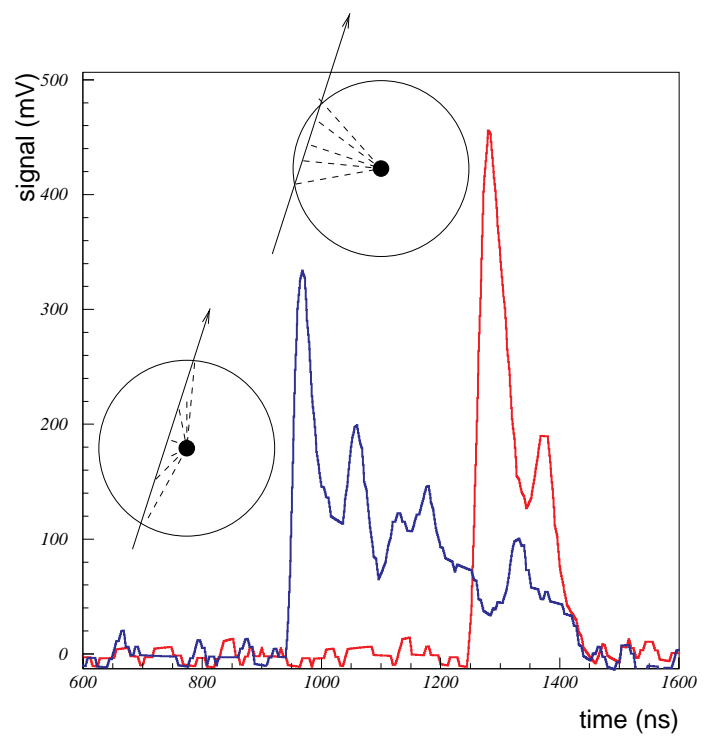

Figure 1: Two typical drift chamber signals. The leading edge measures the distance of the track from the wire. The trailing edge is independent of the track distance and has a fixed latency with respect to the muon crossing time.

\subsection{Leading edge charge}

The difference in spatial resolution for different preamplifier peaking times $t_{p}$ and different discriminator thresholds is mostly due to time-slewing effects, i.e. signal rise time differences from charge fluctuations. The spatial resolution improves for short peaking times and low threshold. Stability against oscillations in a large detector system demands preamp peaking times $t_{p}>10 \mathrm{~ns}$. A lower limit for the discriminator threshold is set by the electronics noise. Typically, the discriminator level can be set to five times the r.m.s. noise level. In well designed systems the noise is dominated by the thermal noise of the termination resistor.

The resolution can be improved with a time-slewing correction by measuring the signal charge within a short gate (gate $<2 \times$ preamp peaking time) following the threshold crossing time. Large pulses shift the threshold crossing to earlier times, whilst small pulses shift it to later times. Knowing the pulse height, a correction for this effect can be applied (Fig. 2).

Apart from this improvement the charge information can also provide very useful information for monitoring purposes (e.g. gas gain monitoring, malfunctioning detector channels, etc.).

\subsection{Two-track separation}

A muon hit may be obscured by a background hit which occurs within one preceding drift time, as the two signals pile up and the muon leading edge is lost. The background signals in ATLAS are caused by shower particles accompanying the muon and uncorrelated signals from high-energy photon background. The inefficiency due to electromagnetic secondaries created by muons in the drift tube wall is $5 \%$. The background rate of $300 \mathrm{kHz}$ raises this inefficiency to $15 \%$.

To reduce the inefficiency, a second discriminator with a high threshold can be used $(\approx 5 \times$ the low threshold) capable of identifying piled-up muon signals. Using such a 


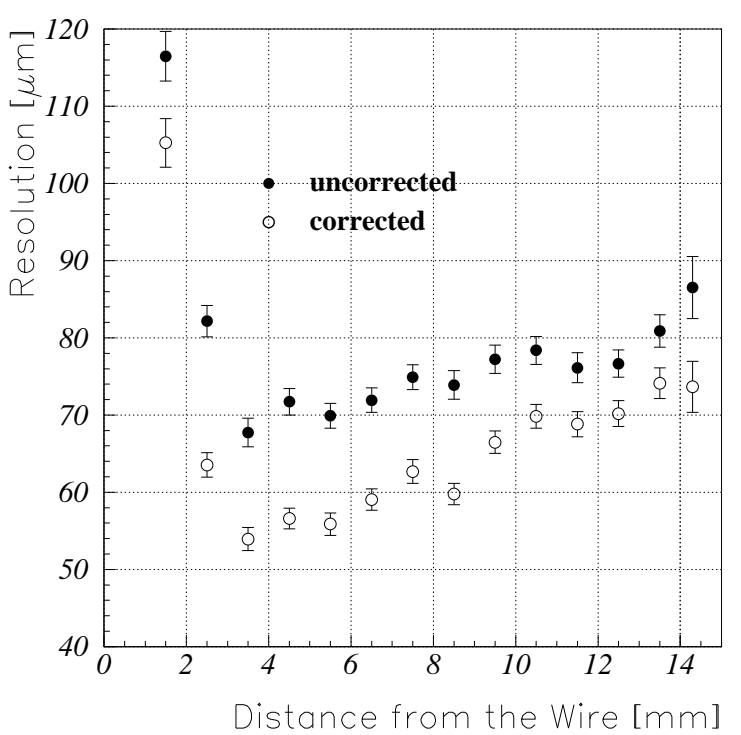

Figure 2: By measuring the signal charge with a short gate ADC we can improve the resolution.

scheme, the probability for identifying a piled-up muon signal is about $70 \%$ if the leading edges of the muon and the background signal are separated by more than $100 \mathrm{~ns}$ (Fig. 3). This reduces the overall inefficiency at $300 \mathrm{kHz}$ background rate to $8 \%$.

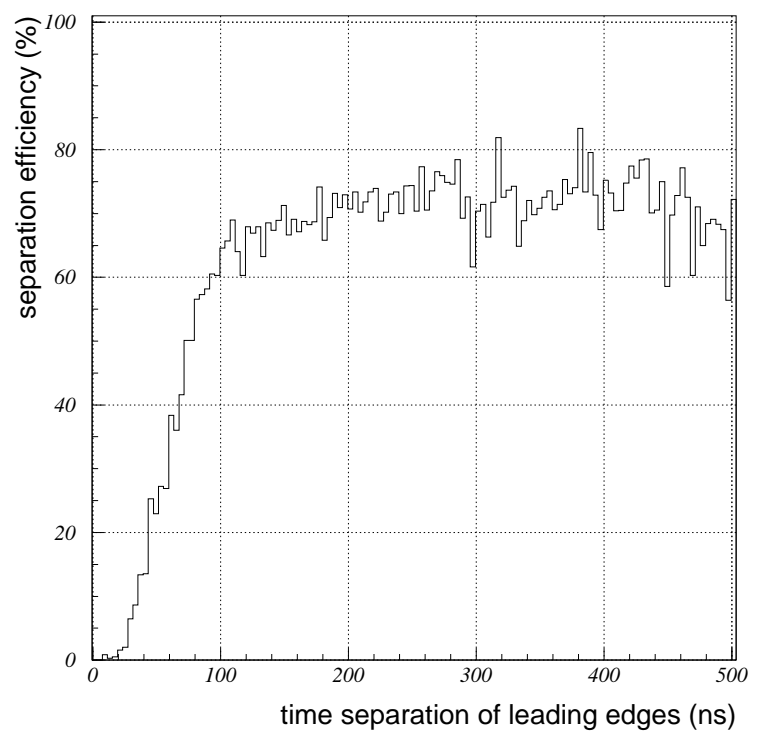

Figure 3: Two-track separation efficiency using a second discriminator with a high threshold.

\section{$3 \quad$ Unipolar versus bipolar shaping}

Baseline fluctuations due to high rates in the ATLAS Muon Spectrometer can be avoided with the use of either bipolar shaping or unipolar shaping with active baseline restoration. Figure 4 shows the same signal for both shaping schemes. Bipolar shaping is a very 'simple' and effective solution but results in multiple hits for single tracks $(2.4$ hits 
on average for the set-up described in Table 1). In addition, the trailing edge information is lost. Using unipolar shaping, the trailing edge information is maintained and we obtain one hit per signal which allows us to encode the multi-hit information into a single output channel, as shown in the next section.

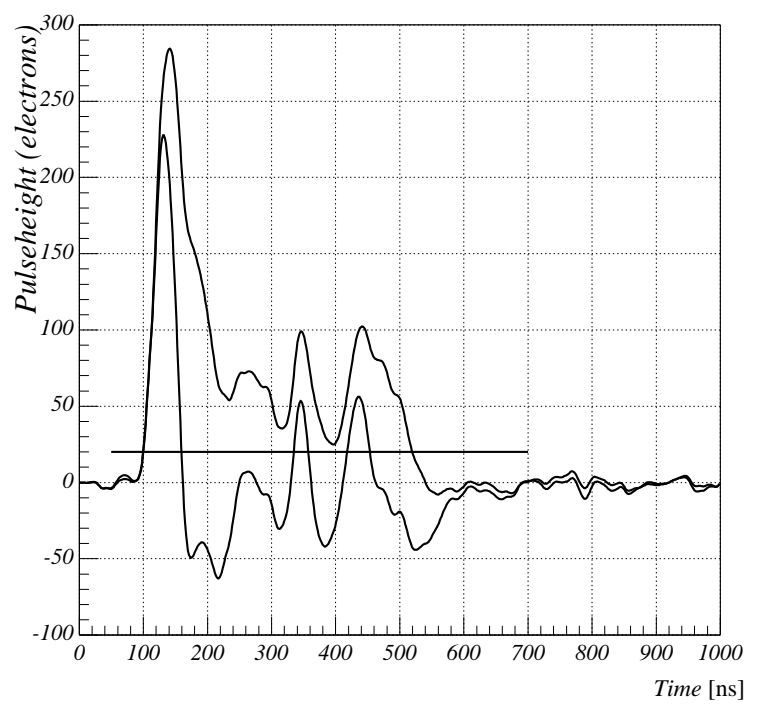

Figure 4: The same signal for a unipolar and a bipolar shaping scheme. The horizontal line indicates the threshold level.

\section{$4 \quad$ Digitization and readout scheme}

The front-end electronics capable of processing this information is shown in Fig. 5. Reading out all the information would require more than one channel per tube. Since only one channel is foreseen a front-end can be designed that provides three different modes of operation.

In the first mode, the leading and trailing edge time of the signal are measured by a time-over-threshold discriminator with the lowest possible threshold. In order to avoid multiple threshold crossing per signal the discriminator has a programmable hysteresis typically set to about half the threshold level.

In the second mode, in addition to the leading edge time, the leading edge charge is measured by a gated integrator controlled by a one-shot which is triggered by the discriminator. The charge contained in the leading edge is stored on a capacitor and then run down in the usual Wilkinson technique and encoded into time information. The gate width is of the order of $15 \mathrm{~ns}$ and the run down time of the order of $100 \mathrm{~ns}$.

The third mode provides leading edge time, charge and multi-hit information. Since the first leading edge of the high threshold discriminator is the same edge already measured by the low threshold discriminator, we can combine the ADC output and the high threshold discriminator output in OR logic. To distinguish hits from the high-level discriminator, the output is given a fixed width of $10 \mathrm{~ns}$ which is always smaller than the ADC output. 


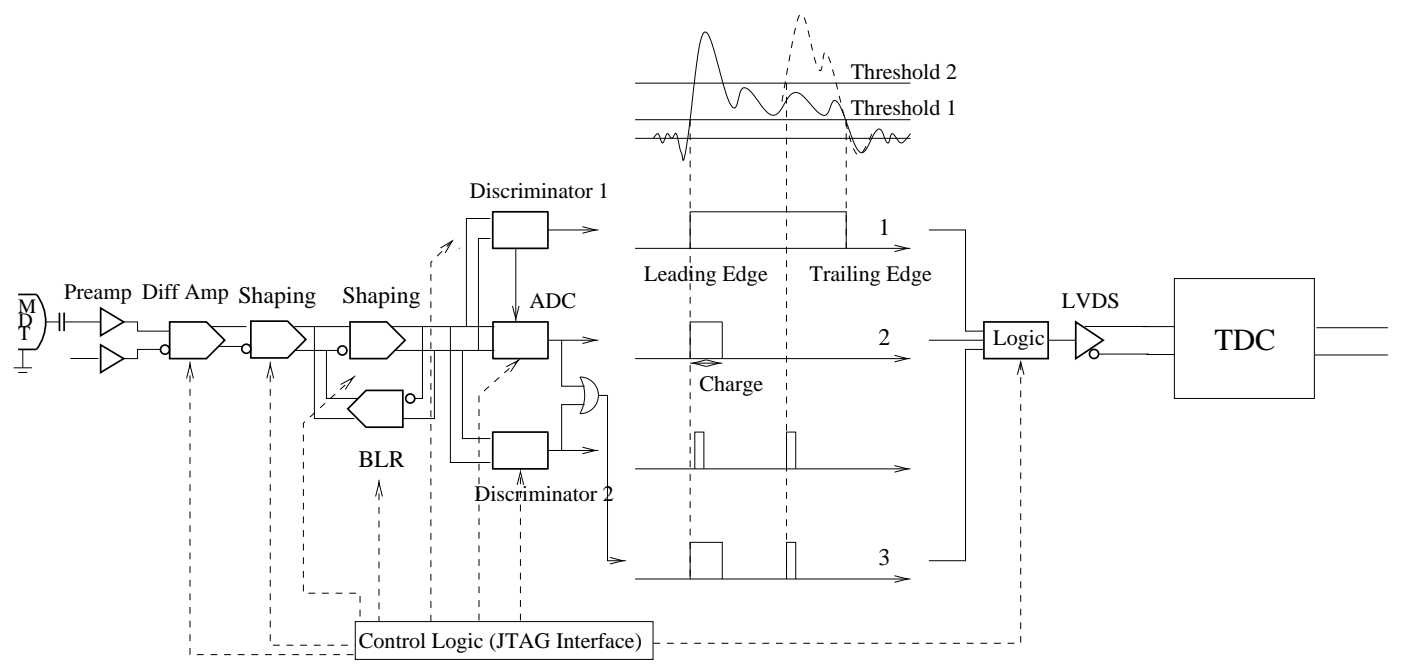

Figure 5: This front-end scheme digitizes all the information contained in the signal and allows three modes of operation to read out specific combinations.

\section{Conclusion}

We have presented a front-end scheme for drift tubes capable of processing several pieces of information contained in the drift tube signal. The leading edge of the signal gives the distance of the track from the wire with an accuracy of $80 \mu \mathrm{m}$. Measuring the leading edge charge of the signal with a short gate ADC we can perform a time-slewing correction and improve the resolution by $15-20 \mu \mathrm{m}$. The trailing edge of the signal has a fixed latency with respect to the bunch crossing and can be measured with a resolution of $20 \mathrm{~ns}$. It can be used to reject out-of-time events. A second discriminator with a high threshold is used to identify piled-up hits with an efficiency of $70 \%$ if the leading edges are separated by $>100 \mathrm{~ns}$. It is possible to choose between different readout options. The system is very economic as all information is encoded in one TDC channel [6].

\section{References}

[1] ATLAS Technical Proposal, CERN/LHCC/94-43, LHCC/P2 (1994).

[2] Nigel Hessey, The precision drift chambers for the ATLAS Muon Spectrometer, Nucl. Instrum. Methods A419 (1998) 326-330.

[3] M. Deile, J. Dubbert, N.P. Hessey et al., Testbeam studies of gas mixtures, CERN ATLAS note MUON-NO 122 (1996).

[4] W. Riegler et al., Resolution Limits of Drift Tubes, Doctoral Thesis (1997).

[5] R.A. Boie, A.T. Hrisoho and P. Rehak, Signal shaping and tail cancellation for gas proportional detectors at high counting rates, Nucl. Instrum. Methods 192 (1982) 365-374.

[6] Y. Arai, J. Christiansen, Requirements and Specifications of the TDC for the ATLAS Precision Muon Tracker, CERN ATLAS note ATL-M-PN-179 (1997). 